\title{
Laboratory-Based Evaluation of SD Bioline HIV/ Syphilis Duo Rapid Test Kits in the Gambia, November 2019
}

J arju ML*, Mendy A, Sanneh ML, Singhateh A, Kijera M, Saho N, J ah FO, J allow K, Ceesay M, J agne S, Baldeh I and Sanneh $B^{*}$

National Public Health Laboratory, Ministry of Health, Bertil Harding Highway, Kotu Layout, Kotu, The Gambia *Corresponding author: Modou Lamin J arju, National Public Health Laboratory, Ministry of Health, Bertil Harding Highway, Kotu Layout, Kotu, The Gambia

Bakary Sanneh, National Public Health Laboratory, Ministry of Health, Bertil Harding Highway, Kotu Layout, Kotu, The Gambia

Received: August 31, 2021; Accepted: October 01, 2021; Published: October 08, 2021

\begin{abstract}
Background: HIV and syphilis are mainly diagnosed using separate rapid test kits for the HIV counseling and testing (HCT), Prevention of Mother-to-Child Transmission of HIV (PMTCT) and other clinical requests in healthcare facilities in The Gambia, as recommended in the HIV testing management guidelines. The goal of this study was to evaluate the performance of SD Bioline HIV/ Syphilis Duo as a single test kit used for the simultaneous detection of HIV \& Syphilis antibodies in the laboratory setting.
\end{abstract}

Methods: We determined the sensitivity, specificity, positive and negative predictive values of SD Bioline HIVISyphilis Duo kits in terms of HIV screening against the GenScreen Ultra HIV Ag-Ab ELISA (Lot 9D0126) using a total of 100 HIV negative and $150 \mathrm{HIV}$ positive serum/plasma specimens that were stored at $-20^{\circ} \mathrm{C}$. Subtyping of the HIV positive specimens was previously performed using First Response HIV-1/2 test cards and Western Blot assays respectively. Moreover, the SD Bioline HIV/Syphilis Duo kit sensitivity, specificity, positive and negative predictive values in relation to Syphilis screening of 100 specimens was also determined against Treponema Pallidum Heamagglutination Assay (TPHA).

Results: In terms of HIV screening ( $n=250)$, the sensitivity, specificity, positive and negative predictive values of the SD Bioline HIV/Syphilis Duo Test Kits were $100 \%, 100 \%, 100 \%$, and $100 \%$ respectively when compared with the GenScreen Ultra HIV Ag-Ab ELISA results in the laboratory setting. Sensitivity for Syphilis screening $(n=100)$ was $95.65 \%$, whilst specificity was $100 \%$ when compared with TPHA. Positive predictive value was $100 \%$, whilst the negative predictive value was $98.86 \%$.

Conclusions: These findings show that the SD Bioline HIV/Syphilis Duo kits have high sensitivity and specificity when used for the detection of HIV and Syphilis antibodies using human serum and/or plasma. Therefore, these kits could serve as a good alternative for the dual elimination of mother-to-child transmission (e-MTCT) HIV and Syphilis through a one-step timely diagnostic procedure, which is crucial in reducing the turnaround time of laboratory test results.

Keywords: HIV; Syphilis; SD Bioline; ELISA; TPHA; Gambia

\section{Introduction}

The number of people living with HIV (PLHIV) globally has reached 38.8 million in 2015. However, HIV/AIDS related mortality has declined from 1.8 million deaths in 2005 , to 1.2 million deaths in 2015 [1]. Worldwide, approximately 930,000 maternal syphilis infections occurred in 2012. These infections caused 350,000 adverse pregnancy outcomes including 143,000 early fetal deaths and stillbirths, 62,000 neonatal deaths, 44,000 preterm or low weight births, and 102,000 infected infants [2]. In Comparison to the 2008 estimates, maternal syphilis decreased by $38 \%$ (from 1,488,394 cases in 2008 to 927,936 cases in 2012) and congenital syphilis decreased by $39 \%$ (from 576,784 to 350,915 ) [2]. Moreover, from 2008 to 2012, the proportion of women not screened for syphilis during pregnancy increased by $49 \%$ in the globe [3]. A review of interventions to screen and manage infections during pregnancy found that those focusing on syphilis demonstrated an $80 \%$ reduction in stillbirths, malaria
(22\% reduction), HIV (7\% reduction) or bacterial vaginosis $(12 \%$ reduction) [3]. It was also found that detection and treatment of syphilis before the third trimester ( 28 weeks) could revert the risk of adverse outcomes to background rates [3]. A rapid assessment of Sexually Transmissible Infections (STIs) in the Gambia found a prevalence of syphilis of $2.8 \%$ in1994 [4]. Another study in 2001 found that syphilis prevalence (7\%) was higher among the 15-34 age group of the sexually active population [5]. Furthermore, a study in 2002 found a $3 \%$ prevalence of syphilis amongst antenatal women [6].

The first case of HIV/AIDS was discovered in The Gambia in 1986. Since then, the HIV epidemic in The Gambia has never gone beyond 5\%. The Demographic and Health Survey (DHS) 2013 results shows that HIV prevalence among adults $15-49$ years in The Gambia is $1.9 \%$ (DHS 2013) [7]. The National Sentinel survey (NSS) among antenatal women showed a declining trend of HIV prevalence from $2.8 \%$ in 2006 to $1.4 \%$ in 2016 [8,9]. However, the Integrated Bio-behavioral
Austin Biomark Diagn - Volume 4 Issue 1 - 2021

Submit your Manuscript | www.austinpublishing group.com

Jariu et al. (C) All rights are reserved
Citation: Jarju ML, Mendy A, Sanneh ML, Singhateh A, Kijera M, Saho N, et al. Laboratory-Based Evaluation of SD Bioline HIVISyphilis Duo Rapid Test Kits in the Gambia, November 2019. Austin Biomark Diagn. 2021; 4(1): 1026. 
study (IBBS) conducted in 2011 have shown a high prevalence (15.9\%) of HIV among female sex workers (FSW) [10]. Such vulnerable groups represent important groups for HIV prevention efforts not only because of their own increased HIV infection rates but because, through their clients that may act as reservoirs for the transmission of HIV, syphilis and other STI to the lower-risk groups in the population of the country [10].

HIV and syphilis are mainly diagnosed using separate rapid test kits for the HIV Counseling and Testing (HCT), Prevention of Mother to Child Transmission of HIV (PMTCT) and other clinical request in The Gambia as recommended in the HIV testing guidelines $[11,12]$. The rapid plasma reagent (RPR) is the rapid test kit for the diagnosis of syphilis even though a study in the country found that it is not very Sensitive (77\%) and not recommended for syphilis diagnosis in poor environmental and resource limited countries [6]. Gliddon HD, et al. 2017 conducted a meta-analysis which included 18 laboratory and Field evaluation studies of diagnostic accuracy for HIV and syphilis and found that SD BIOLINE HIV/Syphilis Duo kit had the best diagnostic performance for screening of HIV and syphilis. The same review showed that dual screening for HIV and syphilis was more cost effective than single rapid tests for HIV and syphilis and prevented more adverse pregnancy outcomes [13]. Furthermore, the use of dual screening of HIV and syphilis as a point of care test was found to be highly acceptable to clients, who cited the quick turnaround time to get results, as well as less cheap services [13]. Studies have shown that SD BIOLINE HIV/Syphilis Duo Test kit is more accurate in the detection of HIV than syphilis [13]. However, most of them were laboratory-based with few field-based diagnostic performances done in resource limited settings [13]. This study intends to evaluate the performance of SD Bioline HIV/Syphilis Duo Test for the rapid testing of HIV and syphilis in the country in the laboratory environment.

\section{Materials and Methods}

\section{Specimens}

The specimens for this study were obtained from banked human serum/plasma harvested from whole blood specimens of patients/ clients who underwent HCT and PMTCT services at health facilities across the country. A total of 350 serum/plasma specimens (100 HIV negative, $50 \mathrm{HIV}-1,50 \mathrm{HIV}-2,50 \mathrm{HIV}-1 / 2$, and 100 specimens for Syphilis screening) were used in this evaluation. These specimens were initially screened for both HIV and Syphilis using separate diagnostic test kits. Storage of the specimens was done at $-20^{\circ} \mathrm{C}$ at the Reference Serology Laboratory of the National Public Health Laboratories (NPHL), The Gambia. Based on the required sample size for this evaluation purpose, specimens were selected at random from the biobank of NPHL, taking into account the integrity of each specimen at the time of selection. Specimens not deemed fit for the purpose of this work were not selected.

\section{Genscreen Ultra HIV Ag-Ab ELISA}

All specimens selected for the evaluation of the SD Bioline HIV/Syphilis Duo test kits were first screened with Genscreen Ultra HIV Ag-Ab ELISA kit (Lot 9D0126) as the gold standard for HIV screening. A specimen distribution and identification plan (worksheet) was first generated followed by the preparation of diluted washing solution and conjugate working solution. Using a precision pipette, $25 \mu \mathrm{L}$ of conjugate 1 (R6) was added in wells that are part of the specimen distribution plan. This was subsequently followed by the addition of $75 \mu \mathrm{L}$ of HIV Ag positive control (R5) to well A1, $75 \mu \mathrm{L}$ of HIV Ab positive control (R4) to well $\mathrm{B} 1,75 \mu \mathrm{L}$ of $\mathrm{HIV}$ negative control (R3) to wells $\mathrm{C} 1, \mathrm{D} 1$ and $\mathrm{E} 1$, and $75 \mu \mathrm{L}$ of each specimen including an in-house control were added to their respective wells as per their positions on the specimen distribution and identification plan. This microplate was sealed with adhesive cover and placed in an incubator for 1 hour at $37^{\circ} \mathrm{C} \pm 1^{\circ} \mathrm{C}$. Following the 1-hour incubation, the adhesive cover was removed and the contents of the plate were washed away with an automated plate washer to get rid of unbound constituents. $100 \mu \mathrm{L}$ of conjugate $2(\mathrm{R} 7 \mathrm{a}+\mathrm{R} 7 \mathrm{~b})$ was dispensed into each well. The microplate was again sealed and incubated for 30 minutes $\left( \pm 4\right.$ minutes) at room temperature $\left(18-30^{\circ} \mathrm{C}\right)$. After this incubation procedure, the microplate was again washed as described above. A substrate solution was prepared and $80 \mu \mathrm{L}$ was dispensed in all the wells. The microplate was subsequently incubated for 30 minutes $\left( \pm 4\right.$ minutes) at room temperature $\left(18-30^{\circ} \mathrm{C}\right)$ followed by the addition of $100 \mu \mathrm{L}$ of stopping solution (R10). The optical density of the microplate was read at $450 \mathrm{~nm}$ using a plate reader. Cut-off values for the tests were determined and results interpreted accordingly. The presence or absence of detectable antigen or antibodies of HIV-1 and/ or HIV-2 was determined by comparing the absorbance measured for each specimen against the calculated cut-off value for the test.

\section{SD Bioline HIVISyphilis Duo test}

The kits were equilibrated at room temperature and the test device removed from the foil pouch. The test devices (cassettes) were labelled with the corresponding patient identification and $10 \mu \mathrm{L}$ of plasma/serum specimen was added into the specimen well of each cassette followed by the addition of three drops of assay diluent to the same well. Test results were interpreted between 15-20 minutes.

\section{Simply Treponema pallidum heamagglutination assay}

Reagents and specimens were placed on the laboratory bench to reach room temperature $\left(15-25^{\circ} \mathrm{C}\right)$. Each serum specimen as well as controls were pre-diluted at 1:20 with Diluent in column 1 (A1-H1) of a microtiter plate (U-shaped bottom). This was followed by the pipetting and dispensing of volumes of $25 \mu \mathrm{L}$ from the pre-dilution to columns 2 and $3(\mathrm{~A} 2-\mathrm{H} 2 \& \mathrm{~A} 3-\mathrm{H} 3)$, which are the test cells and control cells columns respectively. A volume of $75 \mu \mathrm{L}$ of test cells reagent containing chicken erythrocytes coated with Treponema pallidum antigen was added to the test cell column (column 2) and $75 \mu \mathrm{L}$ of control cells reagents was added to the control cells column (column 3). This procedure was subsequently repeated to cater for all the 100 specimens that were to be tested. The microplate was gently mixed to generate a homogenous mixture of the reaction components. A cover was placed on the microplate followed by room temperature incubation $\left(15-25^{\circ} \mathrm{C}\right)$ for 60 minutes. The plate was protected from direct sunlight, and vibrations. Finally, the hemagglutination patterns of the cells was observed and test results were interpreted accordingly.

\section{Data collection and analysis}

Data was collected from HCT and PMTCT laboratory registers at various public health facilities in The Gambia. Statistical analysis was done by using two-by-two tables for calculating sensitivity, specificity, positive and negative predictive values. 


\section{Ethical issues}

In order to ensure patient safety and confidentiality, no individual names or addresses were used to identify the specimens used in this study. Hence, specimens were identified using numbers and/or codes. All the specimens used were obtained from patients/clients who consented to be tested for HIV and Syphilis. These patients/ clients signed consent forms prior to their specimens being collected at public health facilities in the country. As per the country's HIV and Syphilis testing and treatment guidelines/programme, all HIV and Syphilis positive specimens and $10 \%$ of negative specimens are subject to further testing at the National Public Health Laboratories, which is the Reference Centre for HIV and Syphilis testing. As a result, no ethical clearance was needed for this evaluation study.

\section{Results}

\section{Genscreen ultra HIV Ag-Ab ELISA versus SD Bioline HIVI Syphilis Duo}

In a bid to establish the diagnostic accuracy of the SD Bioline HIV/Syphilis kit, a total of 100 specimens that were initially found to be HIV negative on the Genscreen Ultra ELISA also tested negative on the SD Bioline HIV/Syphilis Duo kit. Similarly, 150 specimens that tested positive on Genscreen Ultra HIV Ag-Ab ELISA were also positive/reactive for HIV antibodies using the SD Bioline HIV/ Syphilis Duo kits (Figure 1 and 2).

\section{Treponema Pallidum Heamagglutination Assay versus SD Bioline HIVISyphilis Duo}

For evaluation of the performance of HIV/Syphilis Duo in respect to Syphilis screening, a total of 100 specimens were randomly selected from a pool of previously tested Syphilis specimens. Of these specimens, 22 tested positive on TPHA, whilst as many as 21 of these same specimens tested positive on SD Bioline HIV/Syphilis Duo; with the $22^{\text {nd }}$ samples testing negative.

\section{Discussion}

In this evaluation, the sensitivity, specificity, positive and negative predictive values of the SD Bioline HIV/Syphilis Duo kits in detecting HIV-specific antibodies in human serum and/or plasma was determined by comparing test outcomes with Genscreen Ultra HIV Ag-Ab ELISA using a two-by-two table. Consequently, all the four aforementioned parameters were 100\% (Figure 1 and 2). This shows that the SD Bioline HIV/Syphilis Duo kits could be reliably used in detecting HIV-specific antibodies among individuals being screened for the infection. HIV/Syphilis Duo elucidated very high sensitivity and specificity when used to screen for HIV among pregnant women attending an antenatal clinic East central Uganda [14]. Similar findings were also made when the kits were also used to screen for HIV in pregnant women in Nigeria [15]. These findings are concordant with our findings in this laboratory-based evaluation. The specimens screened were previously subtyped into HIV-1, HIV-2 and HIV-1\&2 using First Response HIV test cards and Western Blot Assays. All 150 specimens that tested HIV positive on ELISA also tested positive on the SD Bioline HIV/Syphilis Duo kits (Figure 2). Furthermore, evaluation of the diagnostic accuracy of the HIV/Syphilis Duo kits with respect to Syphilis screening also showed remarkable performance. Sensitivity and negative predictive value of in screening for Syphilis $(\mathrm{n}=100)$ were $95.65 \%$ and $98.72 \%$ respectively when compared with TPHA (Figure 3). Specificity and positive predictive value were both found to be $100 \%$. Some of these specimens were found to be reactive for both Syphilis and HIV. Similar high diagnostic performance were found in other studies in the past $[14,15]$. It is noteworthy to mention that in this evaluation, the kits were evaluated using HIV-1, HIV-2 and HIV-1\&2 specimens. This was done to ensure that all the HIV types were evaluated on the kit to prevent the occurrence of missed diagnosis when the kit is finally rolled out at various health facilities across the country in the future. Since this evaluation manifests promising results, the utilization of these HIV/Syphilis Duo kit could potentially speed up the dual elimination of HIV and Syphilis especially in the context of the national Prevention of Mother-to-Child Transmission (PMTCT) program.

HIV/AIDS and Syphilis infection still remain a global public threat affecting millions of individuals worldwide. In The Gambia, the dual elimination of mother-to-child transmission (e-MTCT) of

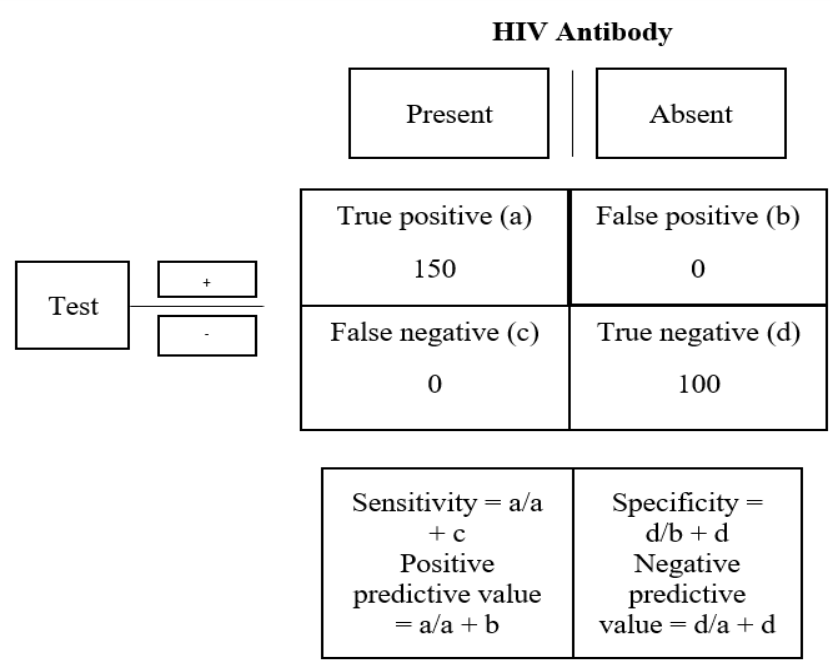

Figure 1: A two-by-two table showing the number of true positive, false positive, true negative and false negative test results used to determine the sensitivity, specificity, positive and negative predictive values of the SD Bioline HIV/Syphilis Duo kits in HIV screening. 


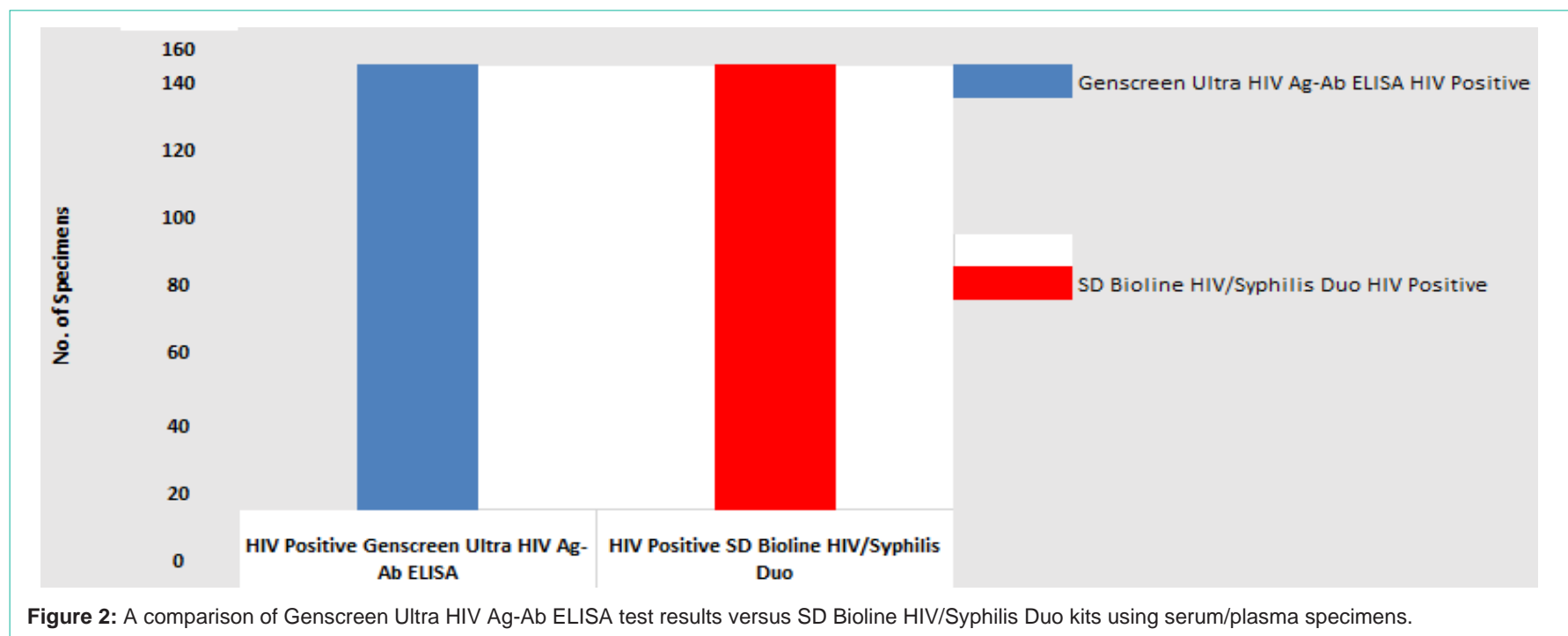

Figure 2: A comparison of Genscreen Ultra HIV Ag-Ab ELISA test results versus SD Bioline HIV/Syphilis Duo kits using serum/plasma specimens.

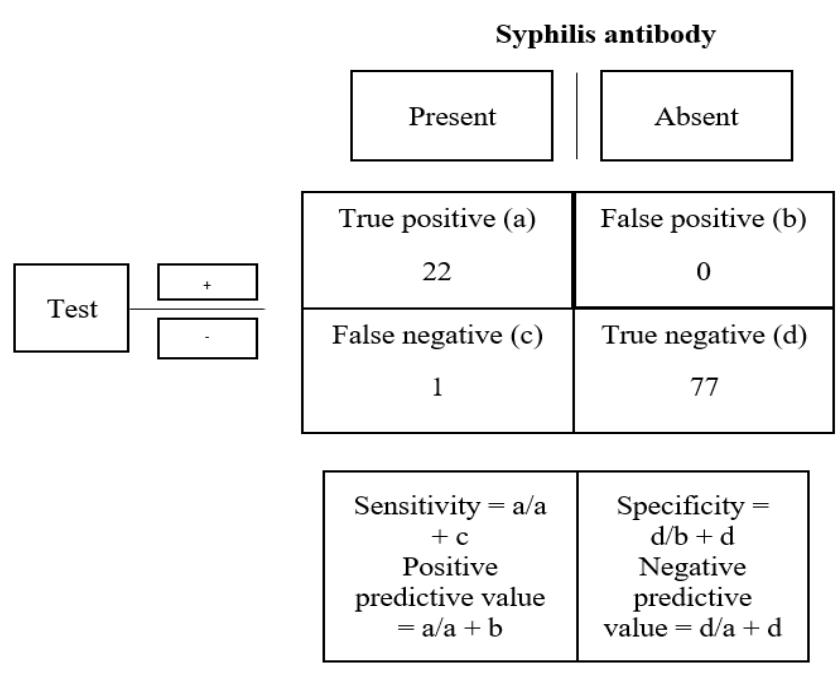

Figure 3: A two-by-two table showing the number of true positive, false positive, true negative and false negative test results used to determine the sensitivity, specificity, positive and negative predictive values in Syphilis screening.

HIV and Syphilis remains a top national health priority. The PMTCT services have been scaled up in the country and HIV screening is well established and efficient. However, syphilis testing is until recently a challenge because the antenatal mothers are mostly tested with Rapid Plasma Reagin (RPR) with very few positive test results at health facilities as was found by West B, et al 2002 [6]. Lately due to this challenge, specimens for Syphilis screening are now occasionally tested using commercially available test cards/strips at health facility level. However, the biggest challenge is the fact that specimens for both HIV and Syphilis screening are currently being tested using two separate test kits in both public and private health facilities across the country. This has led to long turnaround times in obtaining both HIV and Syphilis test results simultaneously from testing sites/ laboratories. In some instances, these delays are due to a shortage of one or both kits (mostly the Syphilis kit) at the time of testing request or due to the heavy workload encountered by the laboratory personnel during a normal working day at a particular health facility. The SD Bioline HIV/Syphilis Duo provides a unique opportunity for the simultaneous detection of HIV and Syphilis in a cost-effective and timely manner.

\section{Limitations of the Study}

The findings of this study do not represent the diagnostic performance of the SD HIV/Syphilis Duo kits using capillary blood, since the specimens used in the study were either serum or EDTA plasma. Moreover, this evaluation was only a laboratory-based one, so field performance was not established.

\section{Conclusion}

The findings of this evaluation shows that the SD Bioline HIV/ Syphilis Duo kits have a high sensitivity and specificity when used for the detection of HIV and Syphilis antibodies using human serum and/or plasma. Thus, these kits could serve as a good catalyst for speeding up the dual elimination of mother-to-child transmission (e-MTCT) of HIV and Syphilis through a one-step, easy to use and timely diagnostic procedure, which is crucial in reducing the waiting 
time of laboratory results. Thus the Gambia had endorsed, procured and implemented the use of SD Bioline HIV/Syphilis Duo kits in the quest to the attainment of the dual elimination of HIV and Syphilis in the country.

\section{Declaration}

Acknowledgements: We would also like to thank the patients and other clients whose stored samples were used for this validation work.

Funding source: We would also like to thank Sangol Farms Gambia Ltd through Abbott Rapid Diagnostic (Ltd) for supporting this study.

Disclaimer: The funders of this work did not influence or interfere in the study design, analysis, report writing and publication of this work.

\section{References}

1. Wang $\mathrm{H}$, Wolock TM, Carter A, Nguyen $\mathrm{G}, \mathrm{Kyu} \mathrm{HH}$, Gakidou $\mathrm{E}$, et al Estimates of global, regional, and national incidence, prevalence, and mortality of HIV, 1980-2015: The Global Burden of Disease Study 2015. The lancet. HIV. 2016; 3: e361-387.

2. Wijesooriya NS, Rochat RW, Kamb ML, Turlapati P, Temmerman M, Broute $\mathrm{N}$, et al. Global burden of maternal and congenital syphilis in 2008 and 2012: a Health systems modelling study. The Lancet Global Health. 2016; 4: e525-e533.

3. Braccio S, Sharland M \& Ladhani SN. Prevention and treatment of motherto-child transmission of syphilis. Current opinion in infectious diseases. 2016; 29: $268-274$.

4. O'Donovan D, Lloyd-Evans N, Manneh K. World Bank Rapid STD assessment in The Gambia. DOSH/MRC. 1994.

5. Shaw Matthew, Marianne Van Der Sande, Beryl West, Katie Paine, Seihou Ceesay, Robin Bailey, et al. "Prevalence of herpes simplex type 2 and syphilis serology among young adults in a rural Gambian community. 2001; 358-365.
6. West B, Walraven G, Morison L, Brouwers J, Bailey R. Performance of the rapid plasma reagin and the rapid syphilis screening tests in the diagnosis of syphilis in field conditions in rural Africa. Sexually transmitted infections. 2002; 78: 282-285

7. GBOS \& ICF. The Gambia Demographic and Health Survey 2013. Banjul, the Gambia and Rockville, Maryland, USA: GBOS and ICF International. 2014.

8. Loeff MF, Sarge-Njie R, Ceesay S, Awasana AA, Jaye P, Sam O, et al. Regional differences in HIV trends in The Gambia: results from sentinel surveillance among pregnant women. Aids. 2003; 17: 1841-1846.

9. HIV prevalence in The Gambia. Data from the antenatal care sentinel surveillance sites 2015-2016. 2016.

10. Mason K, Ketende S, Peitzmeier S, Ceesay N, Diouf D, Loum J, et al. A cross-sectional analysis of population demographics, HIV knowledge and risk behaviors, and prevalence and associations of HIV among men who have sex with men in the Gambia. AIDS research and human retroviruses. 2013; 29: 1547-1552.

11. Ministry of Health \& Social Welfare, Republic of the Gambia. Guidelines for Antiretroviral Therapy for the Prevention and Treatment of HIV in the Gambia. Banjul, The Gambia. 2015.

12. National AIDS Secretariat, Office of the President. "The Republic of the Gambia National Strategic Plan for HIV and AIDS. 2014.

13. Gliddon HD, Peeling RW, Kamb ML, Toskin I, Wi TE, Taylor MM. A systematic review and meta-analysis of studies evaluating the performance and operational characteristics of dual point-of-care tests for HIV and syphilis. Sex Trans Infect, sextrans. 2017.

14. Tarewa IM, Twelwanike A, Mwambi B, Atuhairwe C. Laboratory assessment of SD Bioline HIV/Syphilis Duo Kit among pregnant women attending antenatal clinic Mayuge Health Center III, East central Uganda. 2019.

15. Olugbenga I, Taiwo O, Laverty M, Ngige E, Anyaike C, Bakare R, et al. Clinicbased evaluation study of the diagnostic accuracy of a dual rapid test for the screening of HIV and Syphilis in pregnant women in Nigeria. 2018. 